\title{
An Economic Study of the Efficiency of the Usage of Chemical Fertilizers in the Production of the Most Important Summer Crops in Beheira Governorate
}

Rania A. Elshaer

Senior Res. Agricultural Economics Res. Instit., Agric.Res.Center

$$
\begin{aligned}
& \text { دراسة اقتصادية لكفاءة استخدام الأسمدة الكيماوية فى إنتاج أهم المحاصيل الصيفية فى محافظة البحيرة } \\
& \text { رانيا عبد الفتاح الثناعر } \\
& \text { معهز بحوث الاقتصاد الزراعى - مركز البحوث الزراعية }
\end{aligned}
$$

\begin{abstract}
الملخص

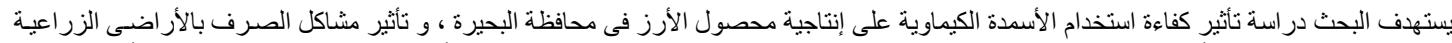

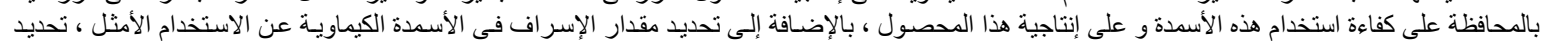

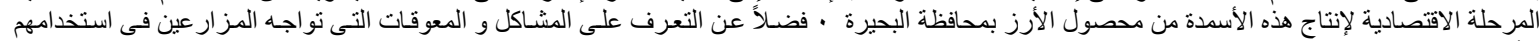

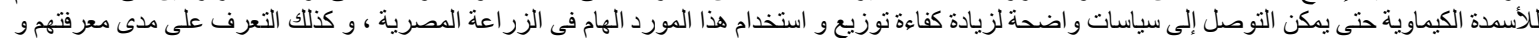

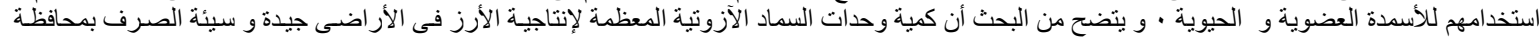

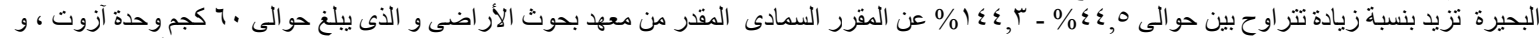

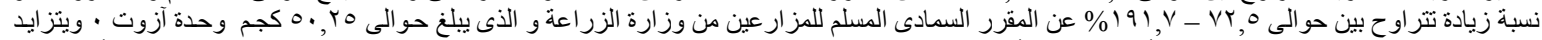

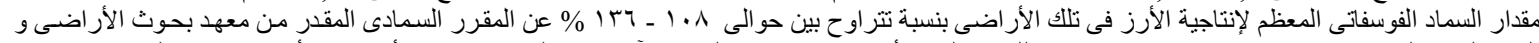

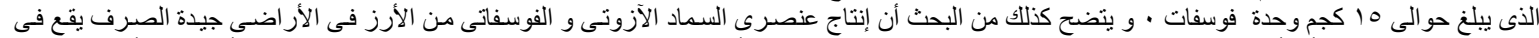

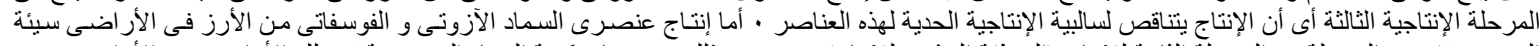

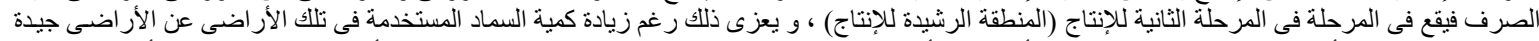

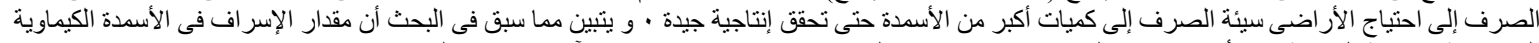

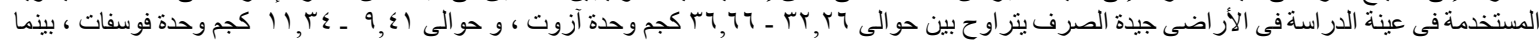

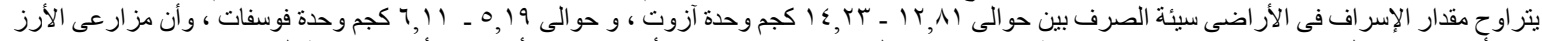

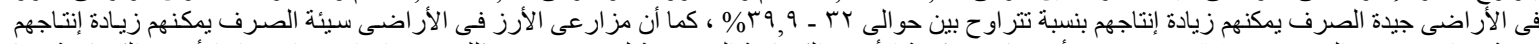

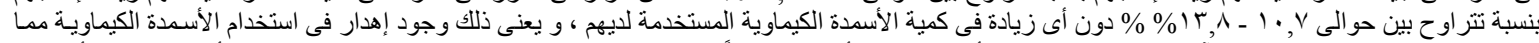

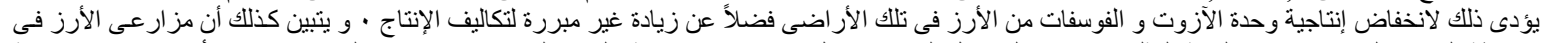

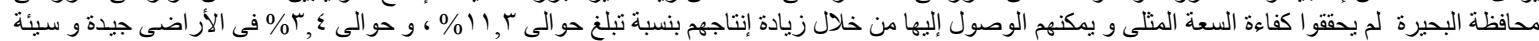

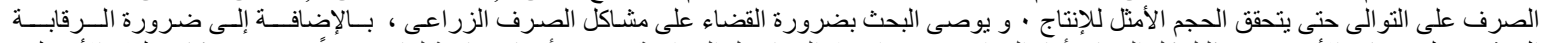

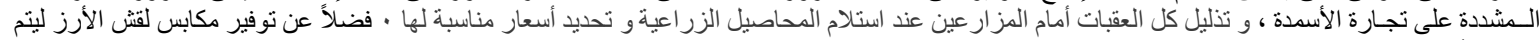

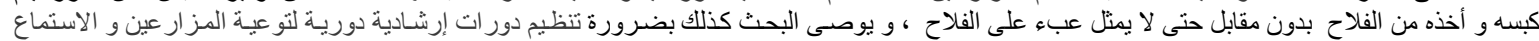

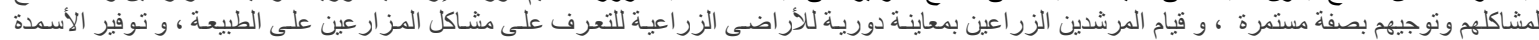

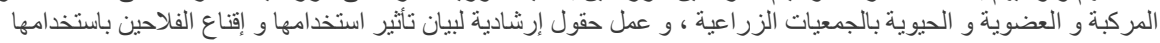

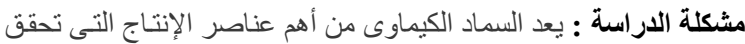

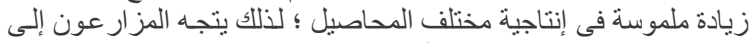

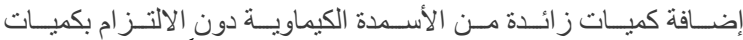

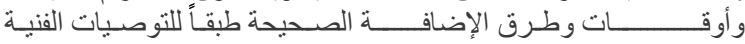

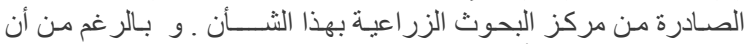

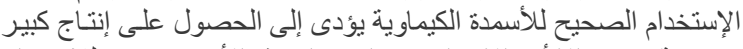

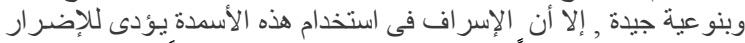

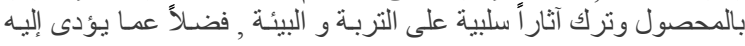

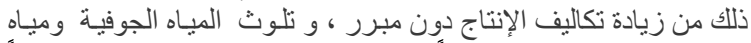

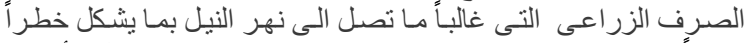

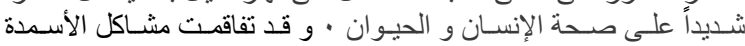

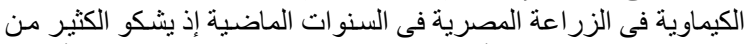

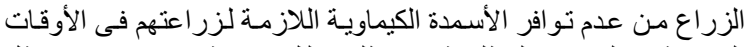

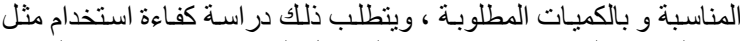

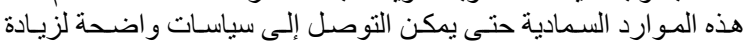

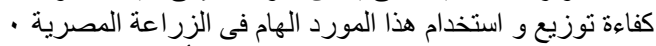

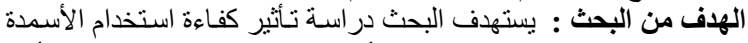

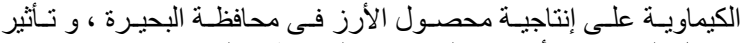

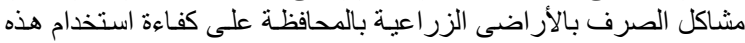

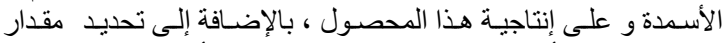

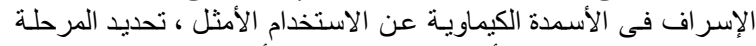

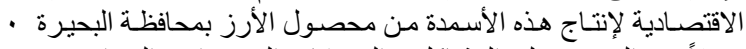

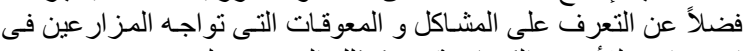

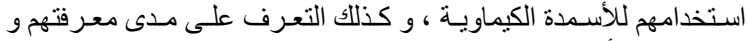

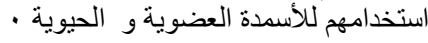
أسلوب البحث و مصادر البيانات

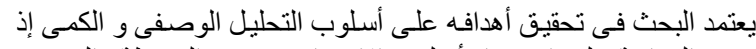

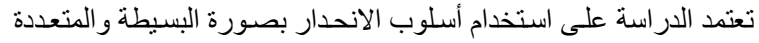

\section{المقدمة}

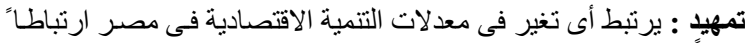

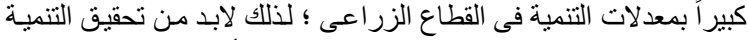

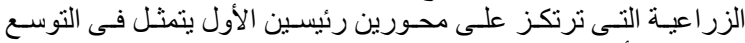

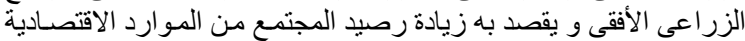

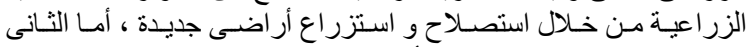

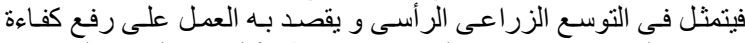

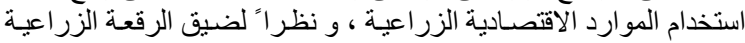

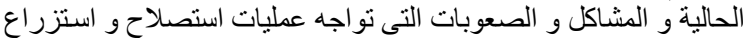

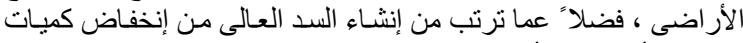

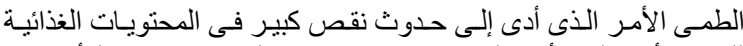

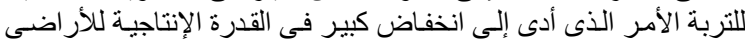

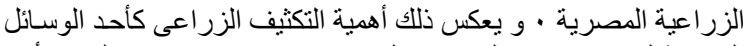

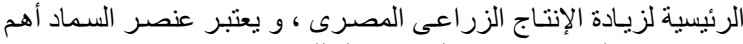

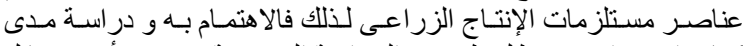

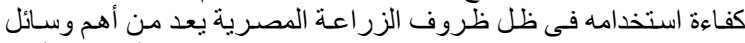

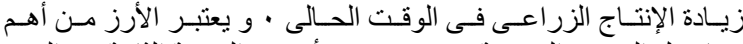

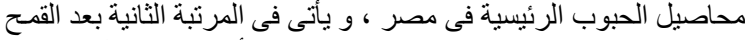

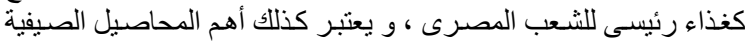

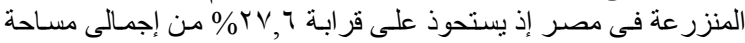

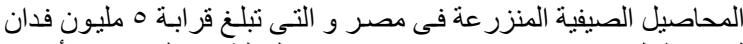

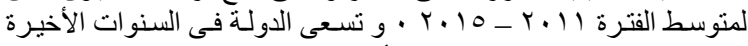

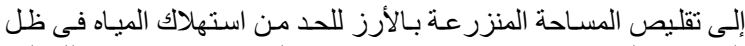

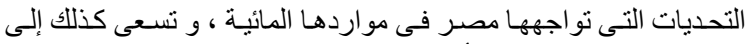

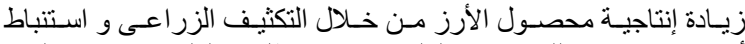

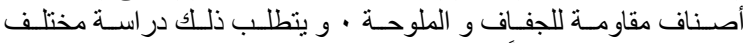
العو امل التى تؤُثر سلباً على إنتاجيـة الأرز حتى يمكن معالجـة أسبابهاو - القضاء عليها 


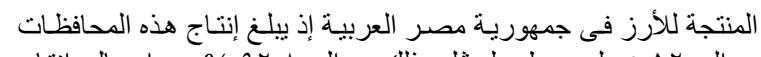

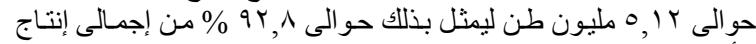

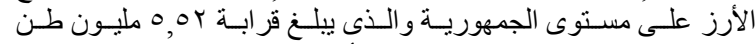

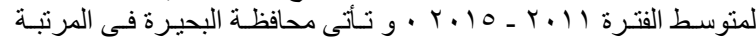

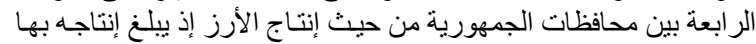

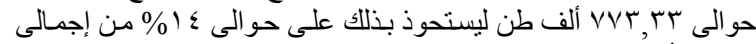

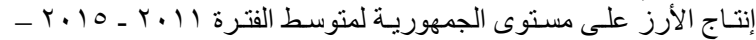

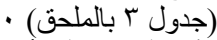
الأهمية النسبية للأرز و تطور إنتاجه فى محافظة البحيرة : يمثل الأرز

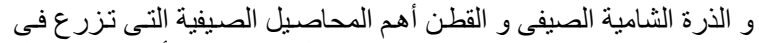

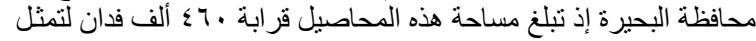

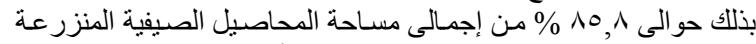

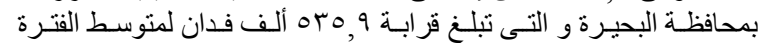

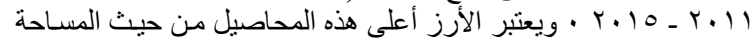

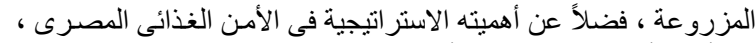

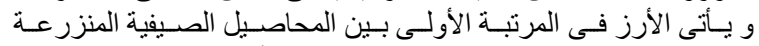

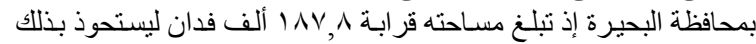

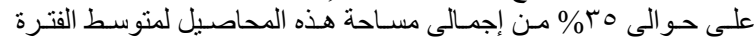

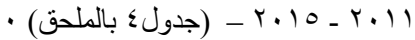

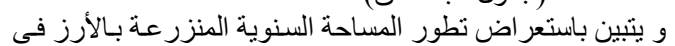

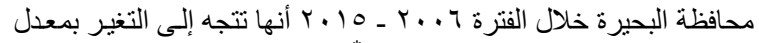

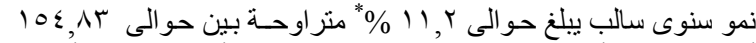

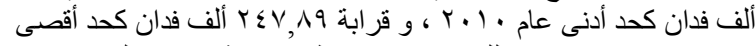

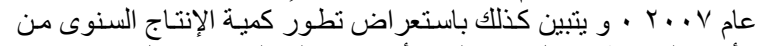

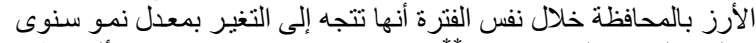

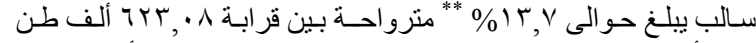

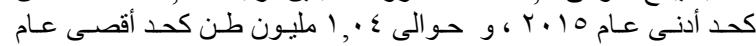

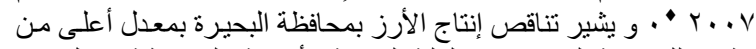

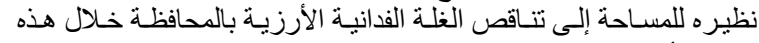

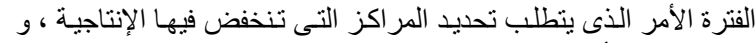

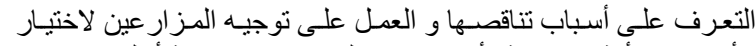

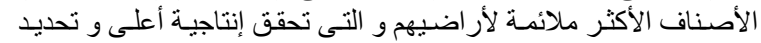

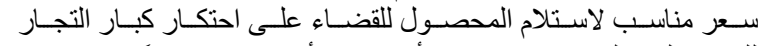

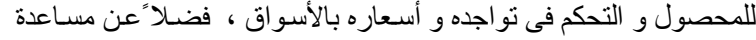

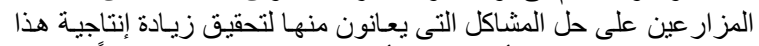

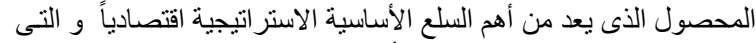

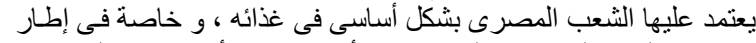

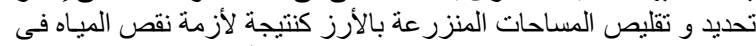

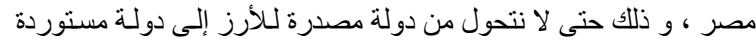

الكفاءة الاقتصـادية لاستخدام الأسمدة الآزوتيـة و الفوسفاتية في إنتاج

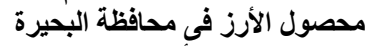

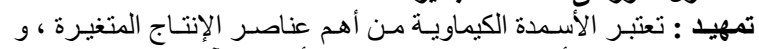

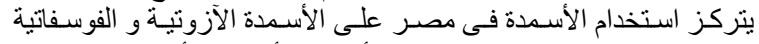

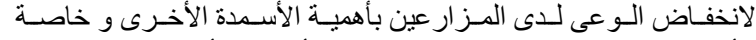

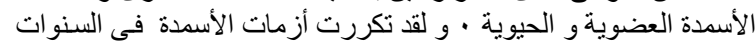

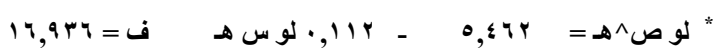

$$
\begin{aligned}
& \left(\&, 110_{-}\right) \quad\left(1 r_{0},\{\wedge q)\right. \\
& \text {, }
\end{aligned}
$$

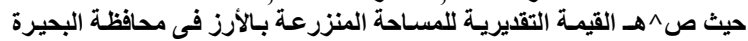

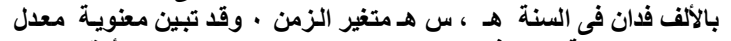

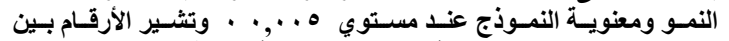

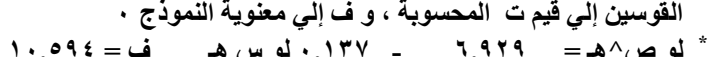$$
(r, r 00-) \quad(99,191)
$$

$\cdot, 014=r-J \quad \cdot, V 00=J$

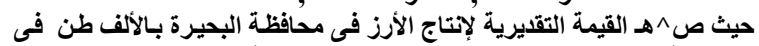

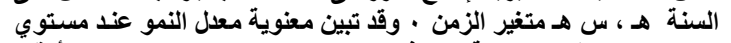

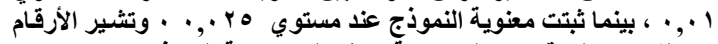

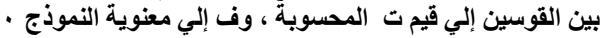

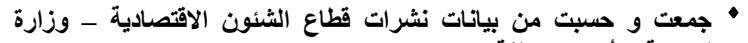

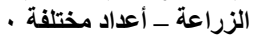

لتقدير الاتجاهات الزمنية العامة لتطور متغيرات الدراسـة ، و لتقدير دالـة التهات

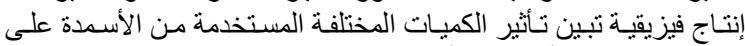

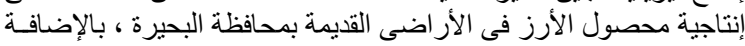

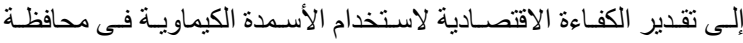

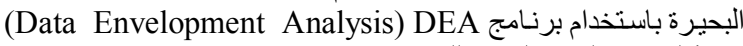

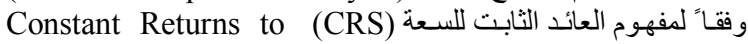
Variable Returns to (VRS) و S Scale

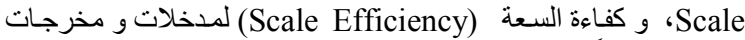

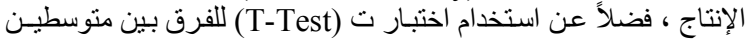

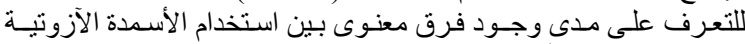

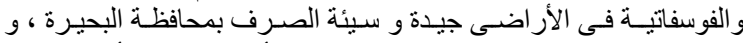

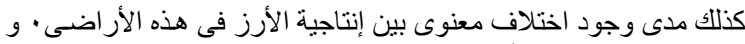

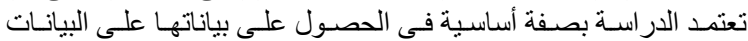

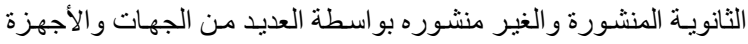

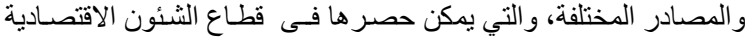

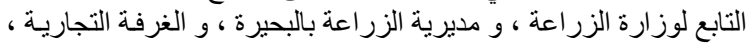

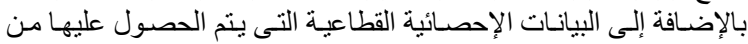

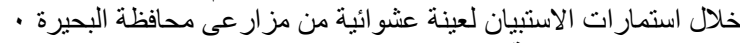

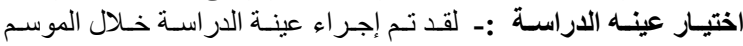

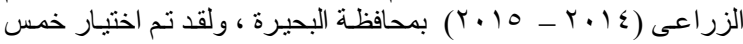

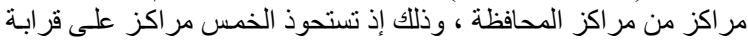

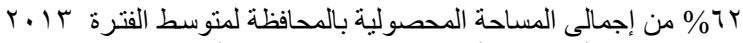

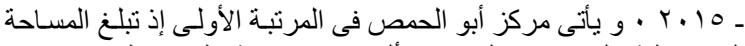

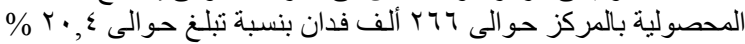

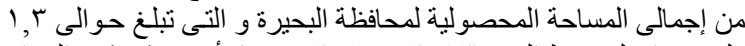

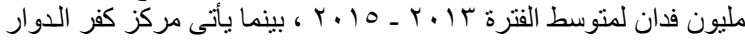

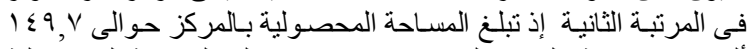

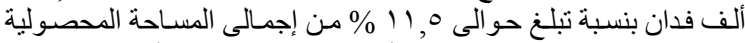

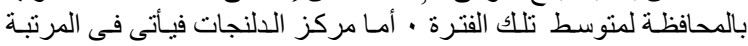

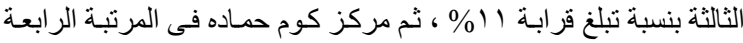

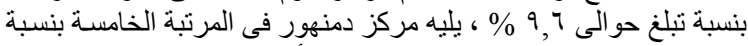

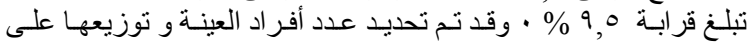

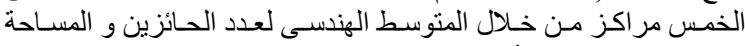

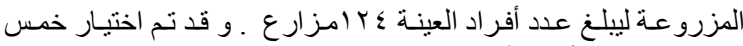

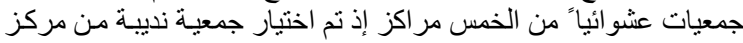

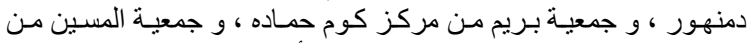

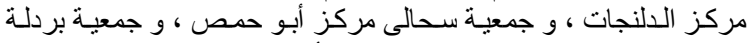

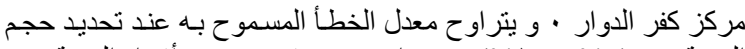

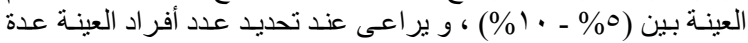

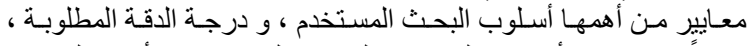

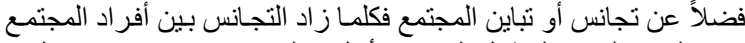

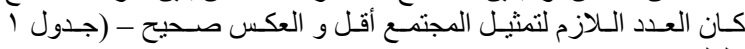

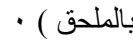

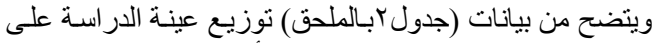

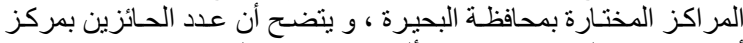

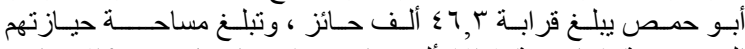

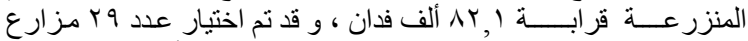

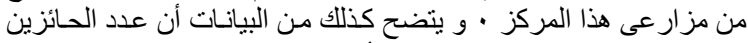

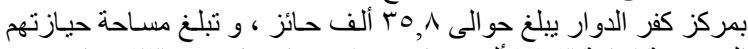

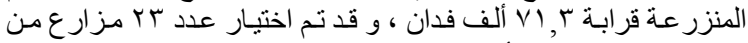

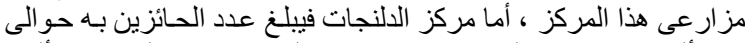

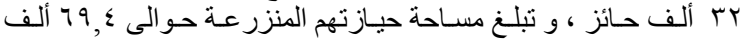

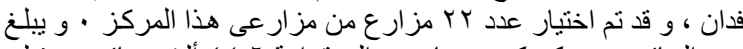

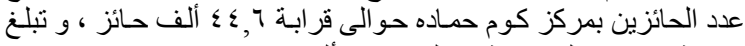

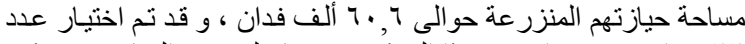

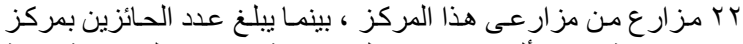

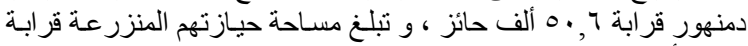

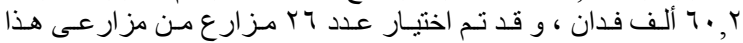

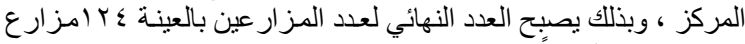

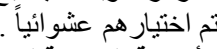

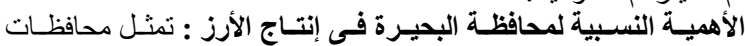

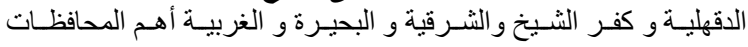


الزر اعة قرابـة هب ــ كجم وحدة آزوت ، و و يزداد بـذلك مقدار السماد

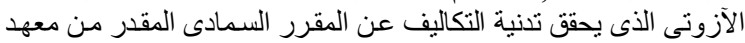

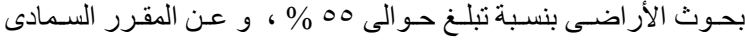

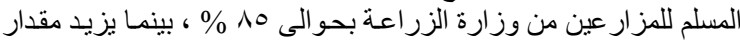

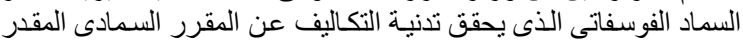

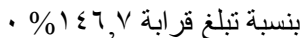

و يتبين كذلك أن كمية وحدات السماد الآزوتيـة المعظمـة لإنتاجية

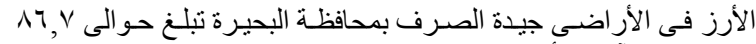

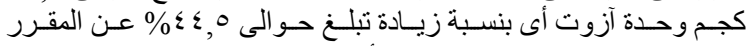

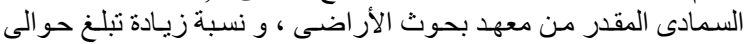

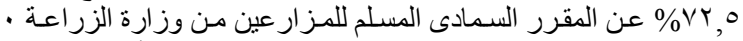

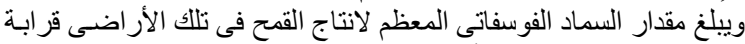

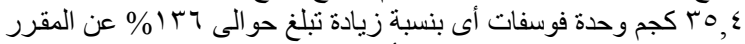

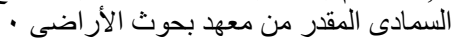

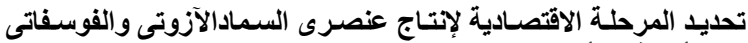

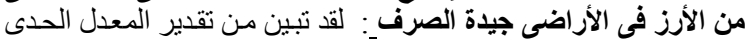

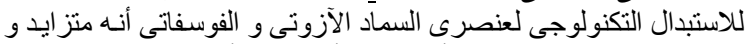

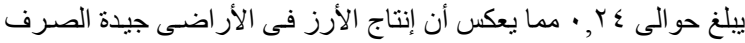

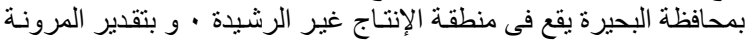

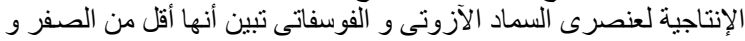

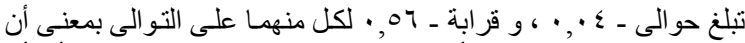

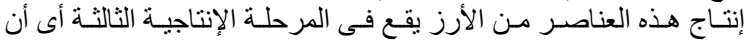

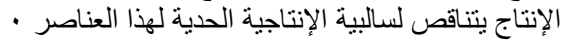

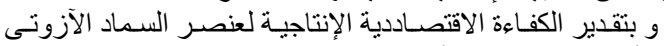

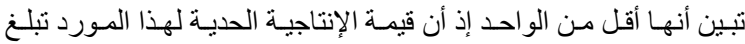

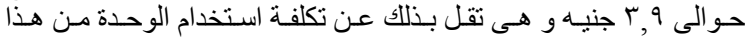

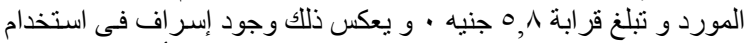

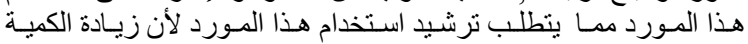

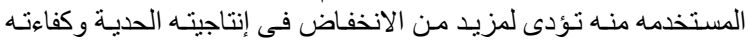

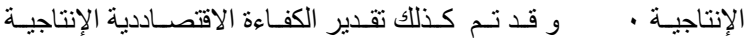

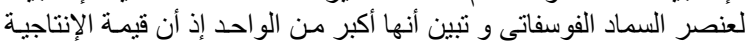

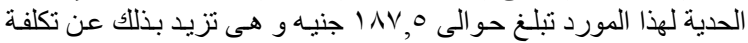

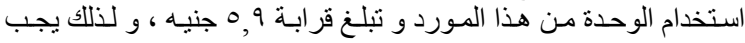

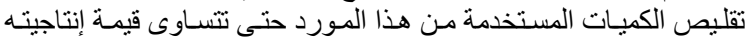

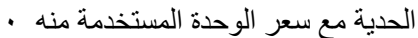

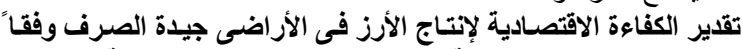

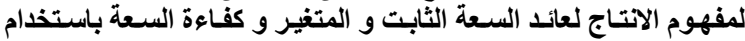

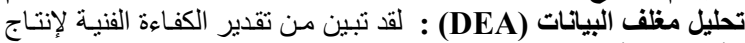

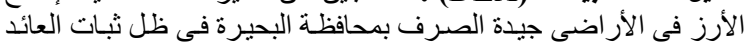

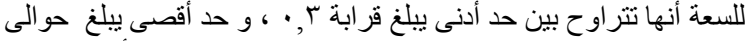

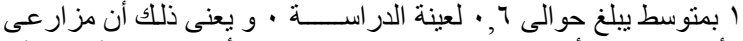

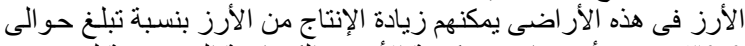

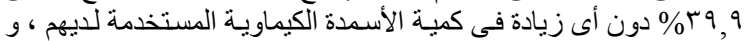

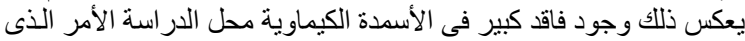

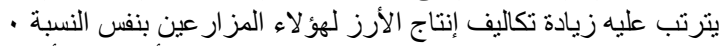

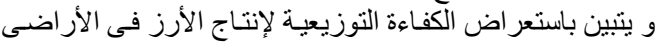

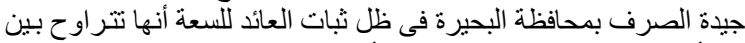

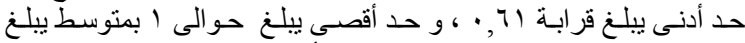

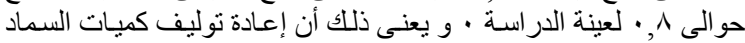

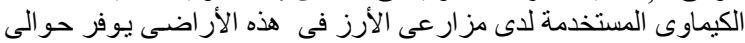

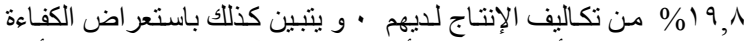

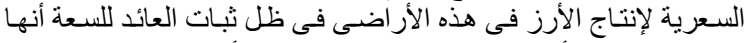

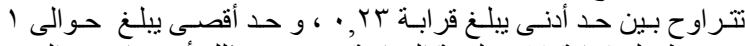

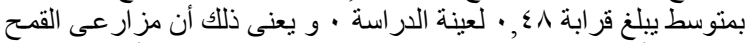

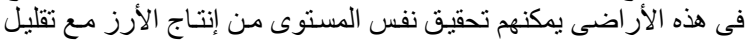

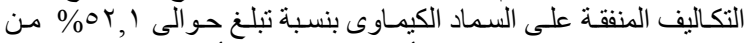

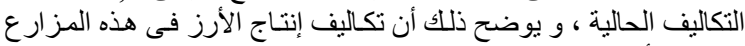

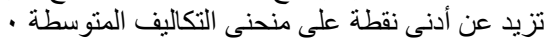

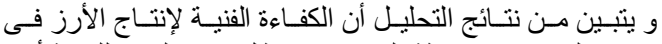

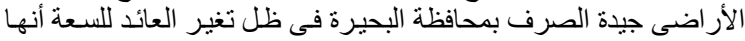

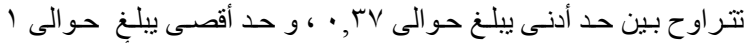

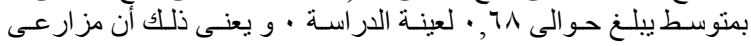

الماضـية سـو اء تمثنل ذلـك فـى نقص المتـو افر بالأسـواق أو الارتفاعـات

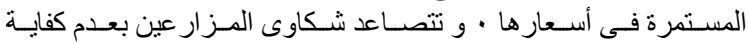

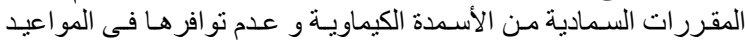

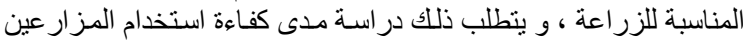

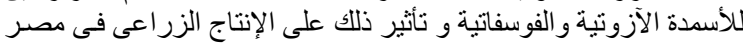

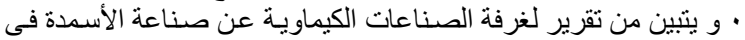

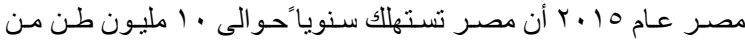

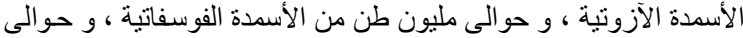

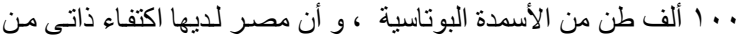

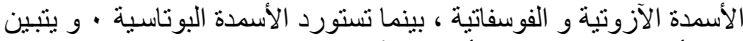

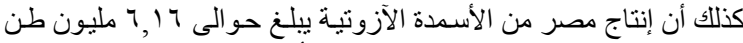

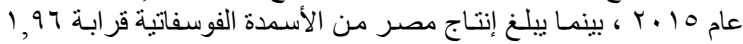

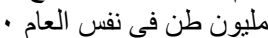

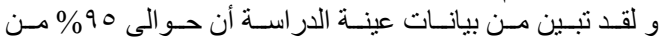

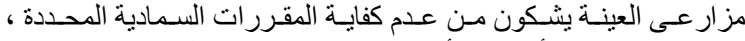

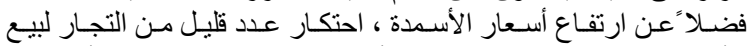

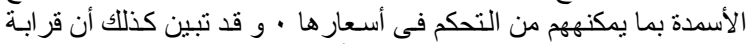

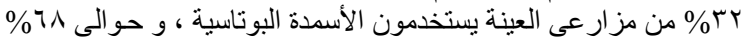

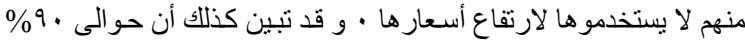

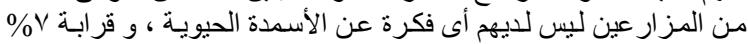

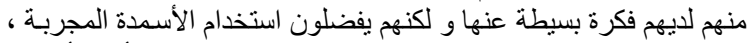

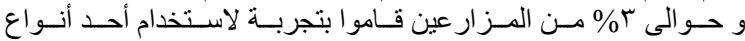

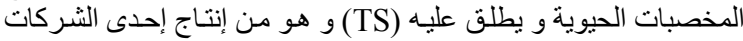

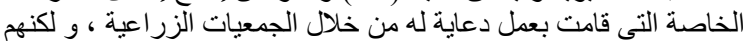

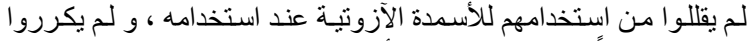

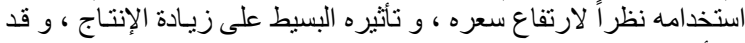

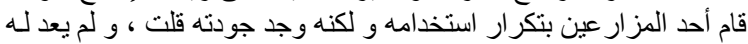

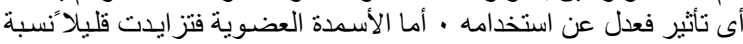

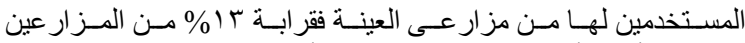

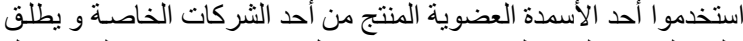

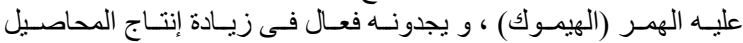

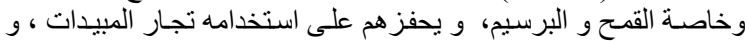

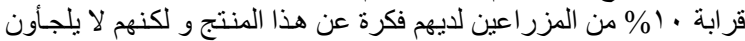

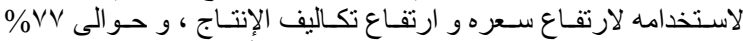

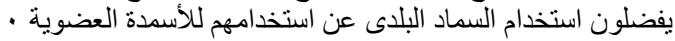

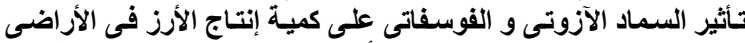

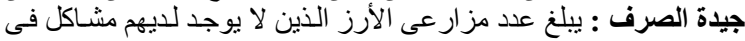

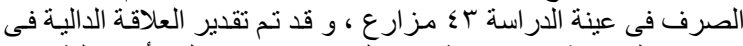

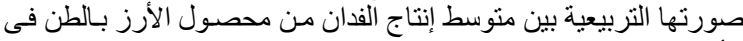

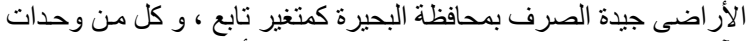

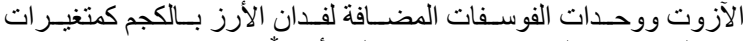

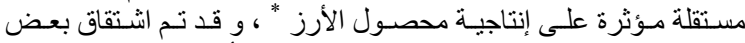

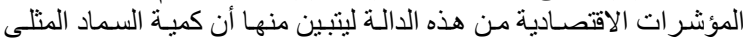

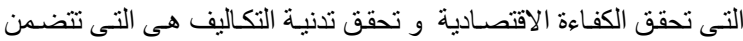

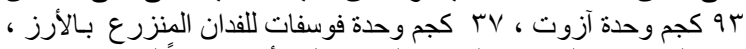

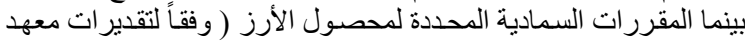

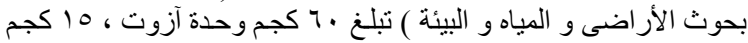

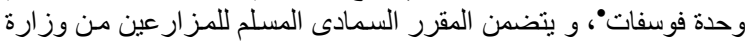

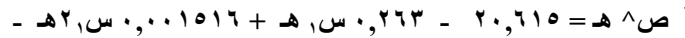
^,

$(1, r Y \varepsilon)(1, V \Psi-)(\varepsilon, \xi \cdot \theta)(\varepsilon, r \mid \leqslant-)(\varepsilon, 1+7)$

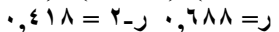

حيث ص^هـ القيمة التقديرية لمتوسط إنتاج الفدان من محصول الأرز بالطن ،

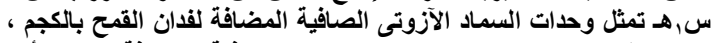

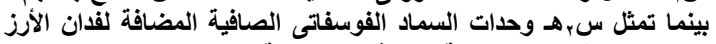

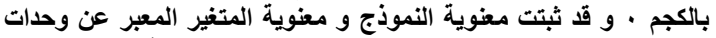

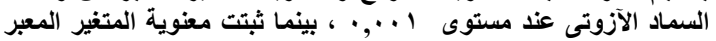

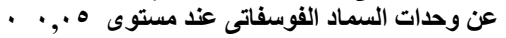

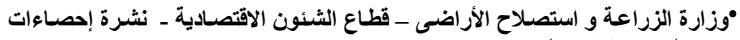

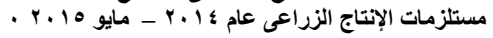




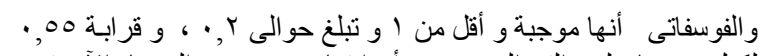

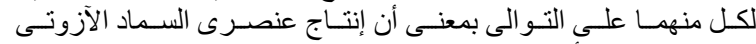

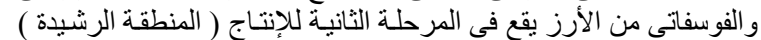

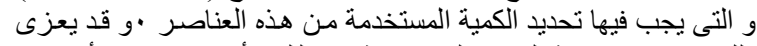

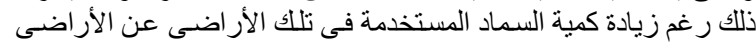

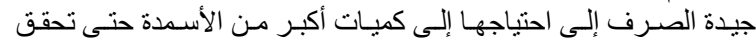
إنتاجية جيدة

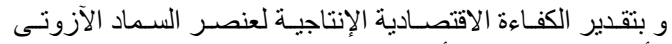

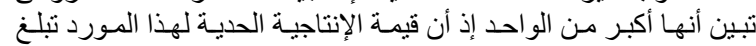

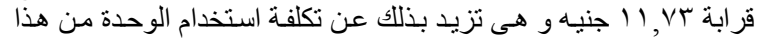

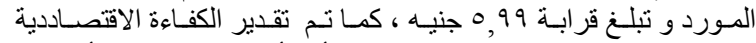

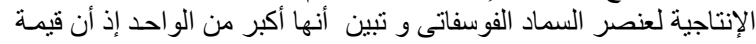

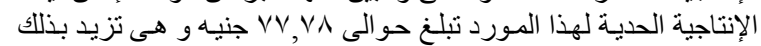

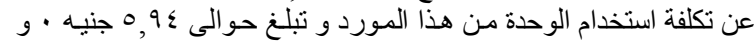

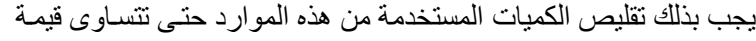

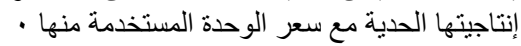

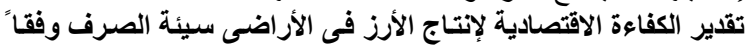

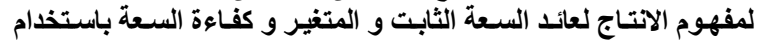

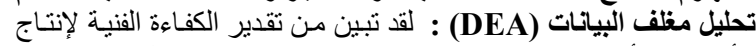

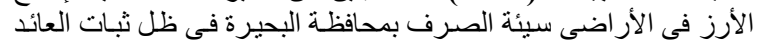

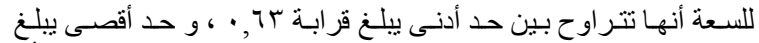

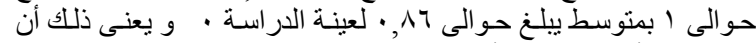

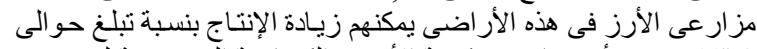

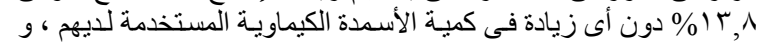

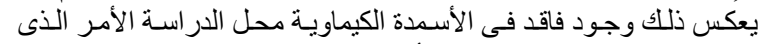

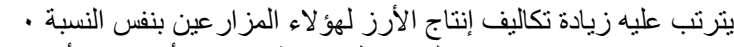

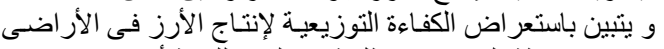

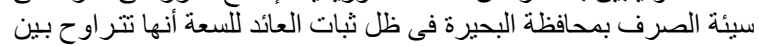

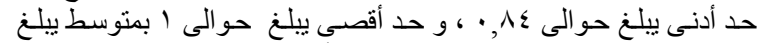

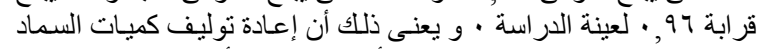

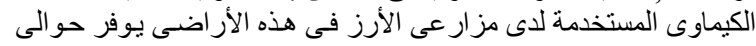

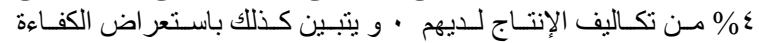

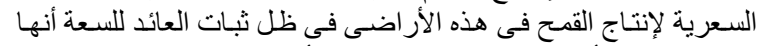

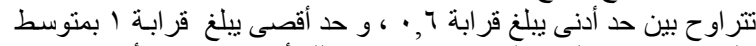

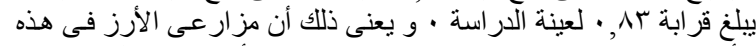

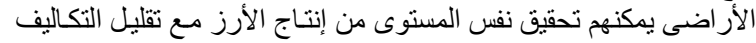

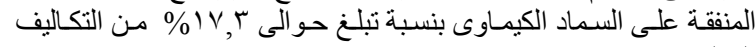
- الحالية

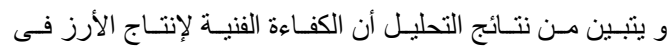

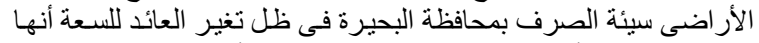

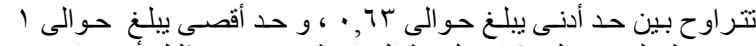

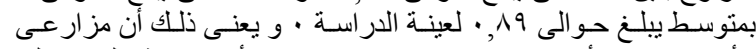

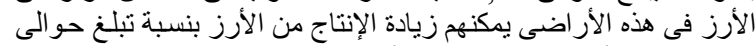

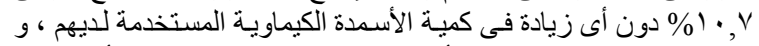

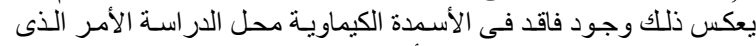

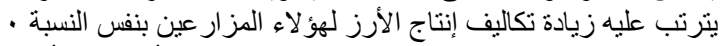

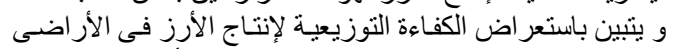

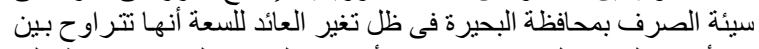

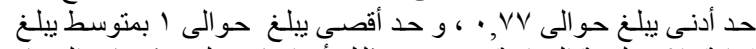

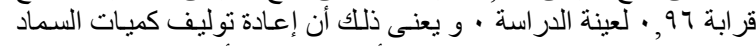

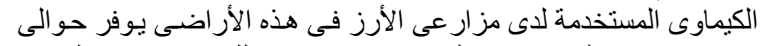

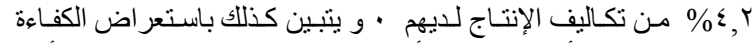

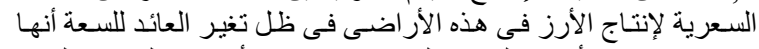

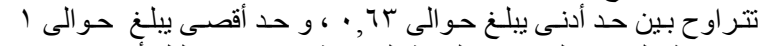

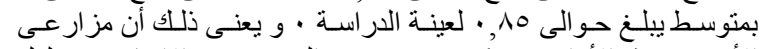

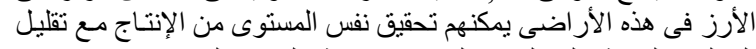

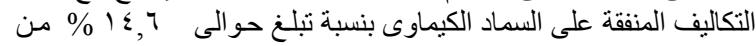

و يتبين من نتائج تقدير مقدار الإسـر اف فـى الأسمدة الكيماويـة

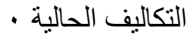

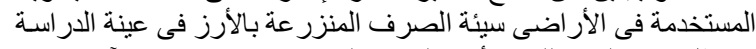

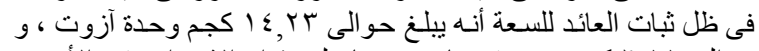

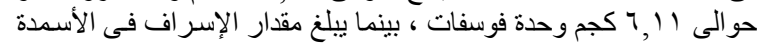

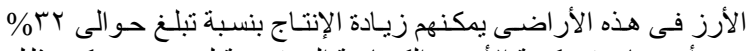

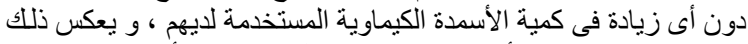

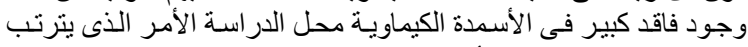

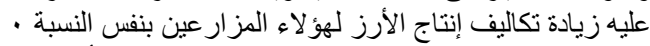

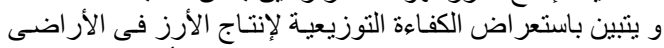

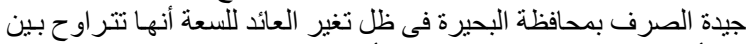

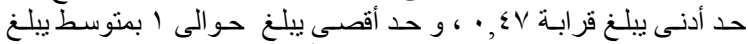

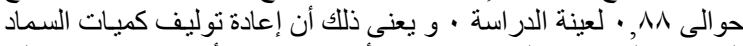

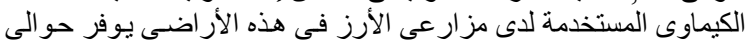

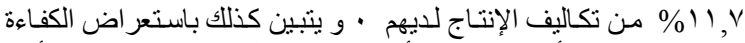

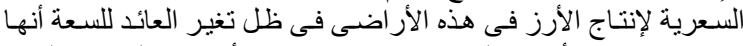

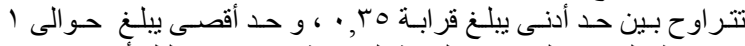

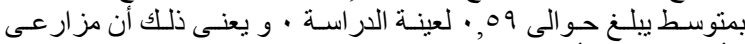

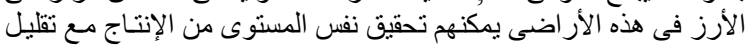

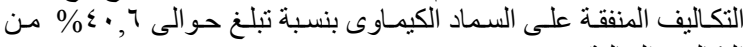

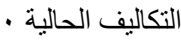

و يتبين من خـلال تقدير مقدار الإسـر اف فـى الأسمدة الكيماويـة

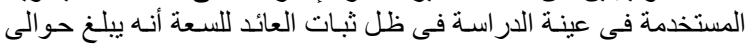

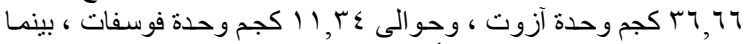

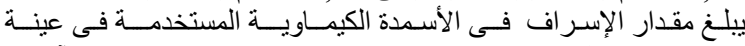

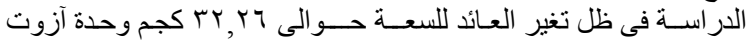

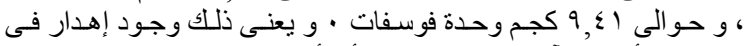

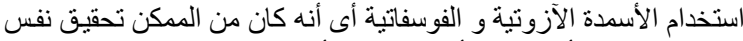

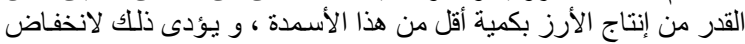

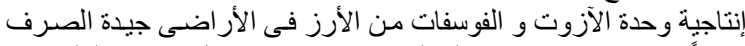

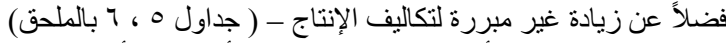

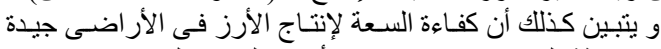

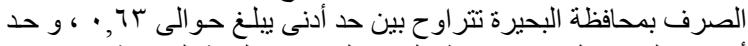

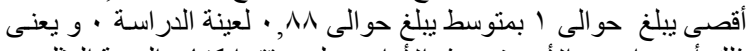

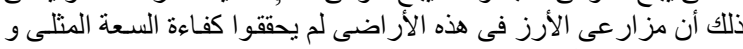

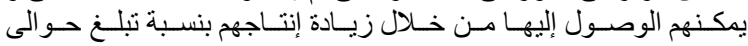
.\% \%

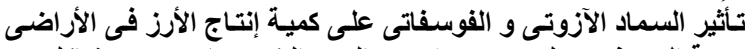

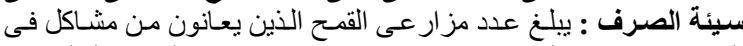

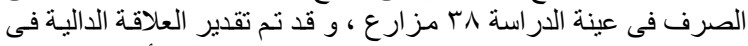

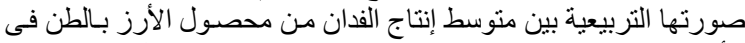

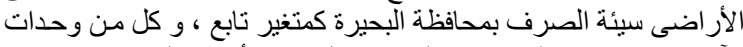

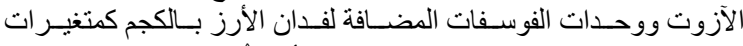

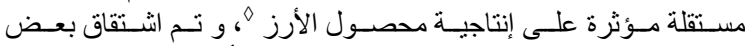

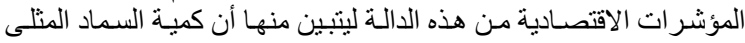

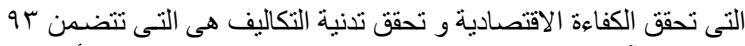

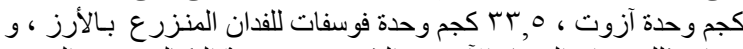

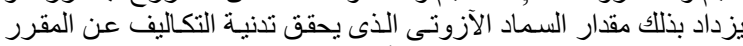

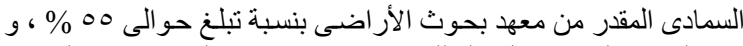

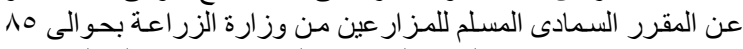

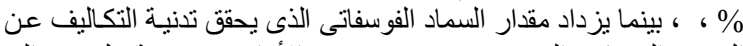

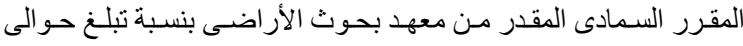

و يتبين كذلك أن كمية وحدات السماد الآزوتية المعظمـة لإنتاجيـة \% \% ITr,

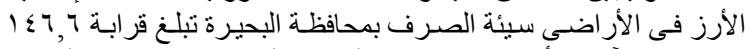

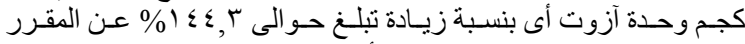

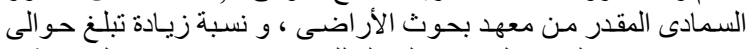

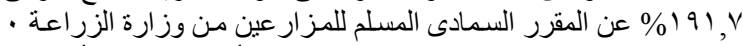

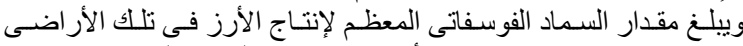

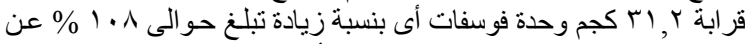

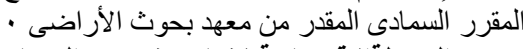

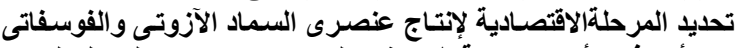

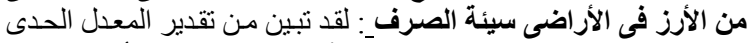

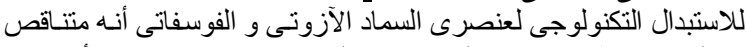

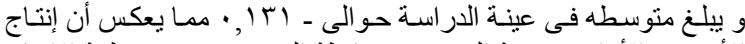

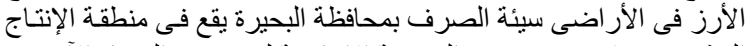
الرشيدة · وقد تبين بتقدير المرونـة الإنتاجية لعنصـرى السماد الآزوتى فئى الإني 


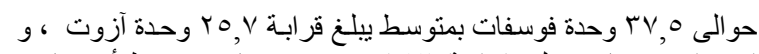

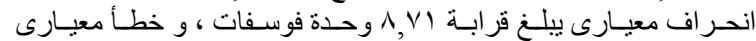

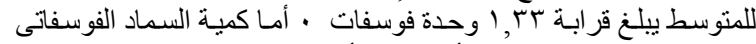

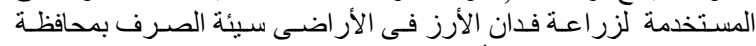

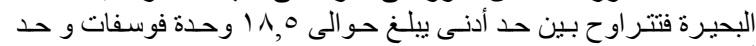

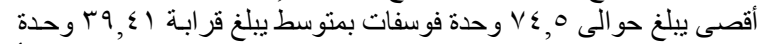

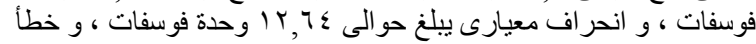

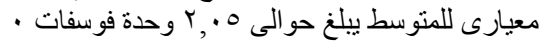

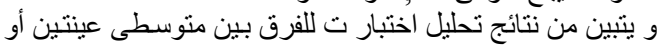

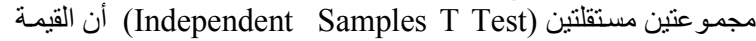

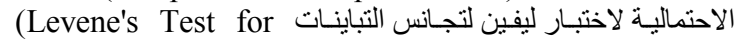
Equality of Variances)

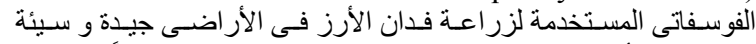

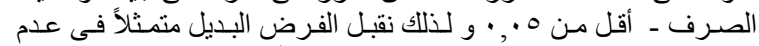

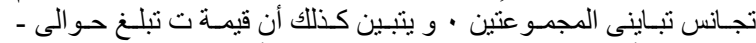

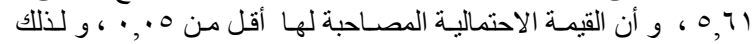

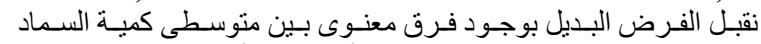

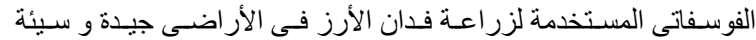

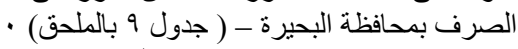

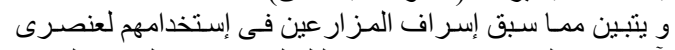

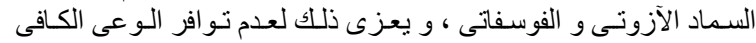

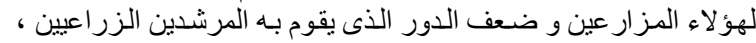

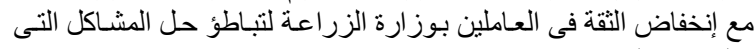

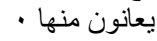

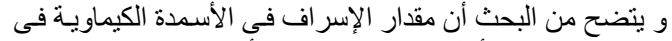

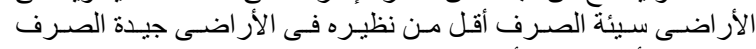

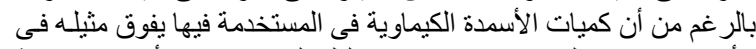

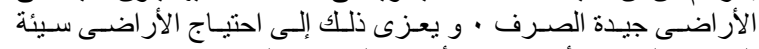

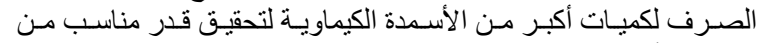

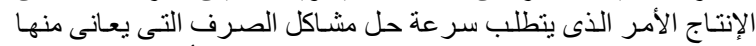

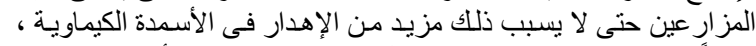

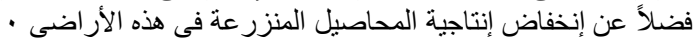

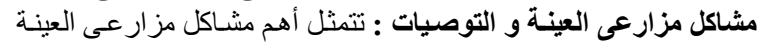

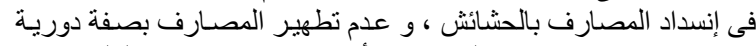

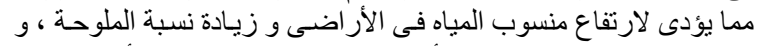

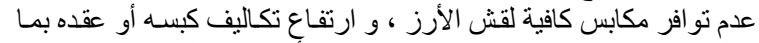

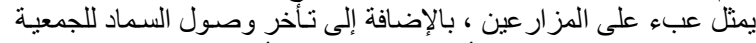

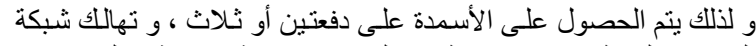

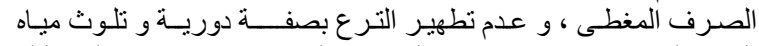

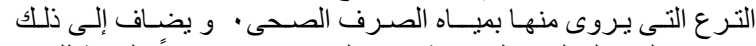

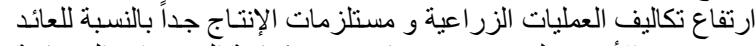

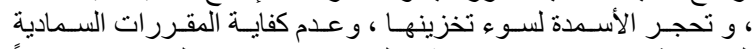

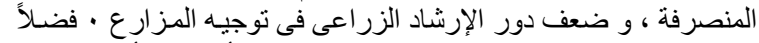

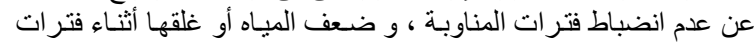

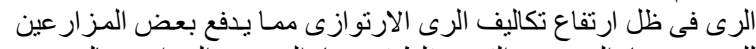

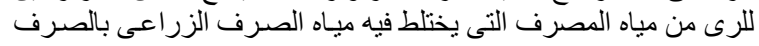

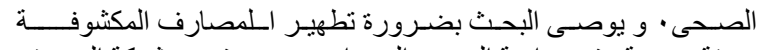

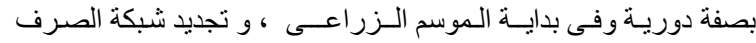

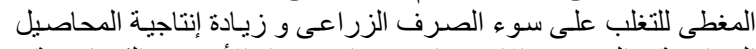

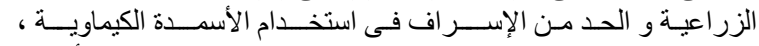

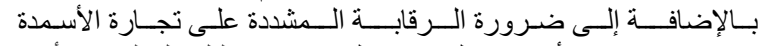

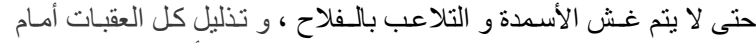

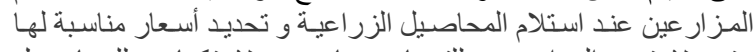

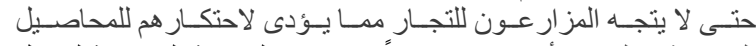

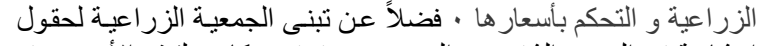

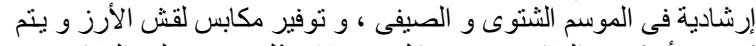

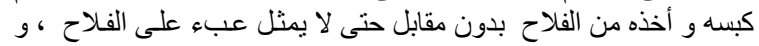

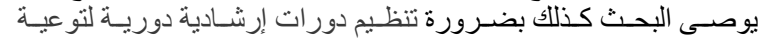

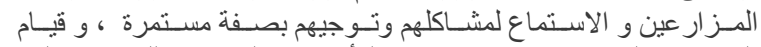

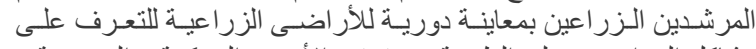

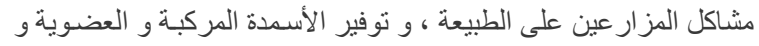

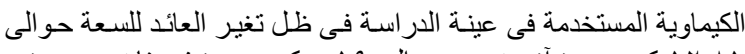

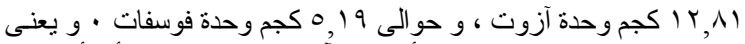

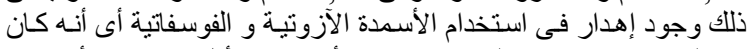

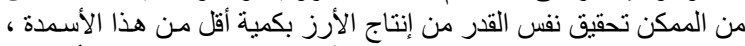

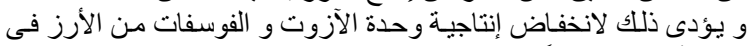

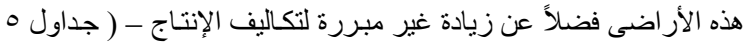

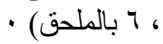

و يتبين كذللك أن كفـاءة السعة لإنتـاج الأرز فى الأر اضسى سيئة

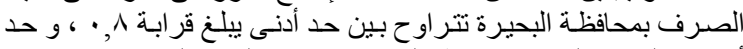

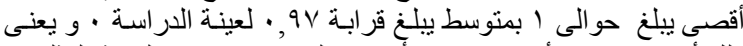

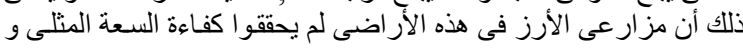

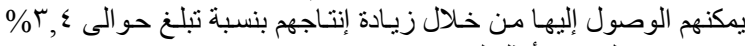

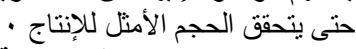

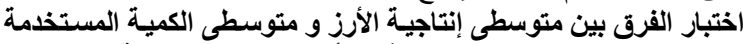

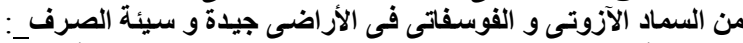

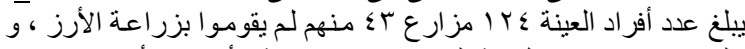

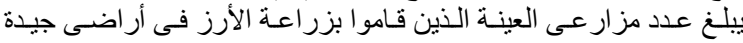

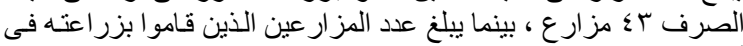

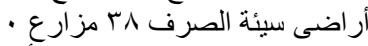

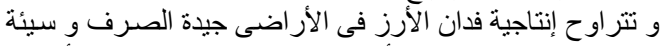

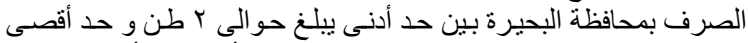

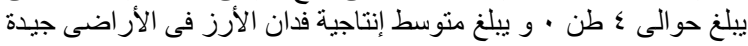

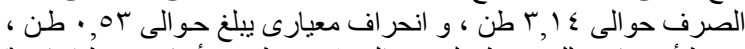

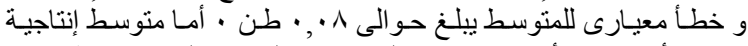

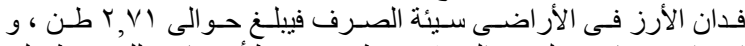

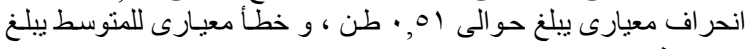

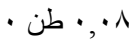

و يتبين من نتائج تحليل اختبار ت للفرق بين متوسطى عينتين أو

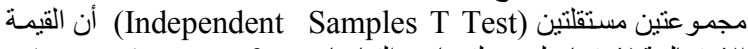

الاحتماليـة لاختبـار ليفين لتجانس التباينـات Levene's Test for Equality of Variances)

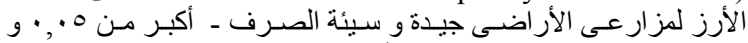

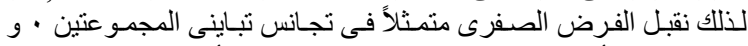

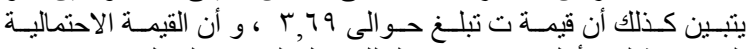

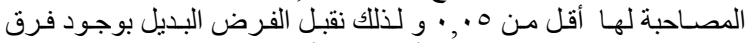

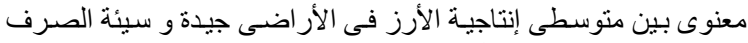

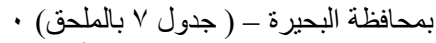

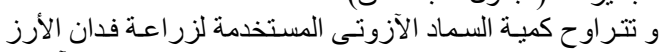

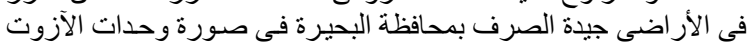

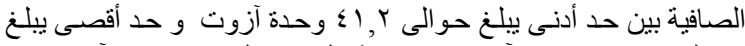

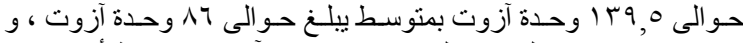

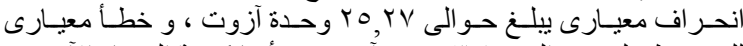

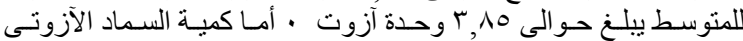

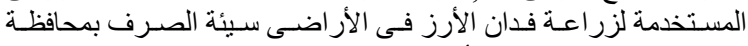

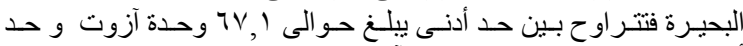

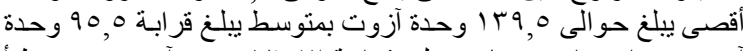

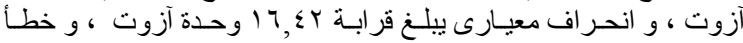

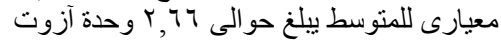

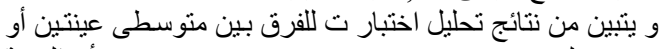

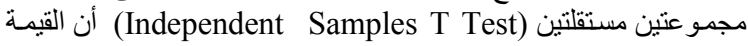

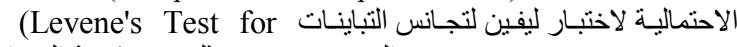
Equality of Variances)

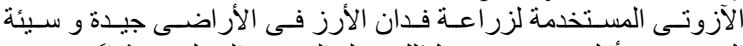

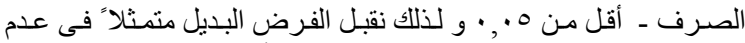

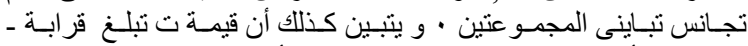

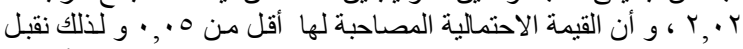

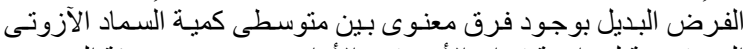

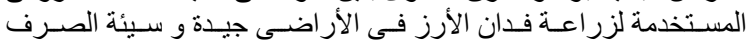

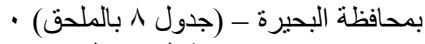

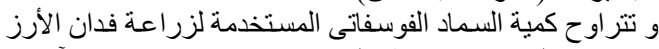

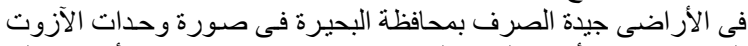

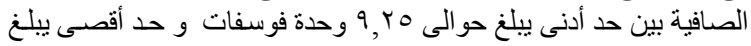


جدول r. . توزيسع عينـة الدراسـة على المراكز المختــارة في محافظة

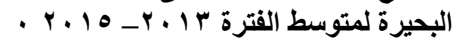

\begin{tabular}{|c|c|c|c|c|c|c|}
\hline \multirow{2}{*}{\multicolumn{2}{|c|}{ الهندسي العزئة العداد }} & \multirow[b]{2}{*}{$\%$} & \multirow{2}{*}{$\begin{array}{c}\text { المساحة المنزرعة الحيازة } \\
\text { بالفدان }\end{array}$} & \multicolumn{2}{|c|}{ عددا لحائزين } & \multirow[b]{2}{*}{ المركز } \\
\hline & & & & $\%$ & العدد & \\
\hline rq & $r T, I T$ & $r r, \wedge 9$ & $\wedge r \cdot 9 r$ & rY,II & STYTT & أبو حمص \\
\hline rT & $11,9 V$ & $r \cdot,{ }_{0}$ & VIYAE & IV,IY & $r 0 \wedge \leq r$ & كفر الدوار \\
\hline YY & $I V, V$. & $r \cdot, r)$ & $79 \leq Y T$ & $10, r 1$ & TY. & الدلنجات \\
\hline$r \varepsilon$ & $19,0$. & $1 V, T \leqslant$ & $7.71 \leq$ & $r, r^{\prime}$ & $\leqslant \varepsilon 07 \Lambda$ & كوم حماده \\
\hline rq & $r \cdot, r \cdot$ & $1 V, 01$ & 7.17. & $r \leq, 1 V$ & 0.091 & دمنهور \\
\hline$K \varepsilon$ & $1 \ldots$ & $1 \ldots$ & $r \leqslant 117 \Lambda$ & $1 \ldots$ & $r .94 \cdot 1$ & الاجمالى \\
\hline
\end{tabular}

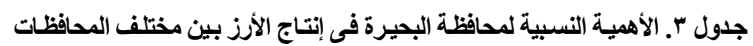

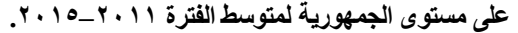

البيانظة

الدقهلية

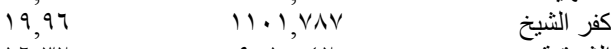

الثرقية الثغ

البحيرة

$9,07 \quad$ orv, الغربية

محافظات أخرى

إلجمالى الجمهورية أخرى

المصدر: جمعت و حسبت من بيانات نشرات قطاع الثشئون الاقتصادية ــ أعداد مختلفة ـ

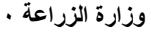

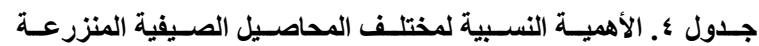

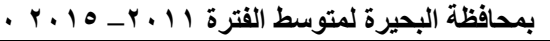

\begin{tabular}{|c|c|c|}
\hline الأهمية النسبية & المساحة بالألف & 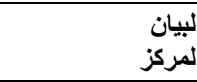 \\
\hline$r_{0}, \varepsilon \varepsilon$ & $|A V, V V|$ & j \\
\hline$r \varepsilon, \cdot r$ & IAY,rYT & رة الثامية الصيفى \\
\hline $17, V V$ & $\wedge q, \wedge \vee 1$ & طن \\
\hline $1 \leq, 17$ & $\vee \circ, \wedge \wedge \leq$ & لاصيل أخرى \\
\hline $1 \ldots$ & Oro, 10 & ساحة المحصولية \\
\hline
\end{tabular}

المصدر: جمعت و حسبت من بياتات نثرات قطاع الثنئون الاقتصادية ـ أعداد مختلفة -

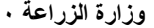

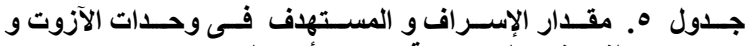

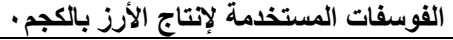

\begin{tabular}{|c|c|c|c|c|c|c|}
\hline \multicolumn{3}{|c|}{ ( في ظل ثبات العائد للسعة الصرة) } & \multicolumn{3}{|c|}{ 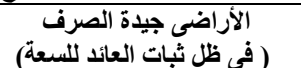 } & \multirow[t]{2}{*}{ السماد } \\
\hline الإسراف & المستهـف & الفُعلى & الإسراف & المستهرف & الفعلى & \\
\hline $1 \leqslant, Y \mu$ & NI,YT & $90, \leqslant 7$ & 47,77 & $\varepsilon q, r \leqslant$ & NT & يونة بون \\
\hline 7,11 & rT, & $r q, \leqslant 1$ & $11, r \varepsilon$ & $1 \leqslant, r_{0}$ & $r 0,79$ & س \\
\hline
\end{tabular}

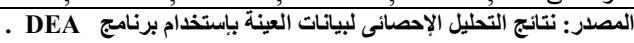

جدول 1 ـ مقدار الإسراف و المستهذف فى وحدات الآزوت و الفوسفات

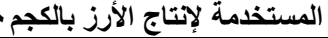

\begin{tabular}{|c|c|c|c|c|c|c|}
\hline & & & & & & \\
\hline للسعة & كي سيئة العائد الص & & د للسعة & ضل تغي جيدة اللئ & & السماد \\
\hline الإسراف & المستهذف & الفعلى & الإسراف & المستهدف & الفُطى & \\
\hline$|r, \lambda|$ & Nr,To & $90, \leqslant 7$ & $r Y, Y T$ & or,,$\leqslant$ & $\Lambda T$ & الآزوتى \\
\hline 0,19 & $T \leqslant, Y T$ & $r q, \leqslant 1$ & $9, \leqslant 1$ & $17, Y 1$ & Yo,79 & الفو سفاتى \\
\hline
\end{tabular}

جدول V. اختبار ت للفرق بين متوسطى إنتاجية محصول الأرز فى

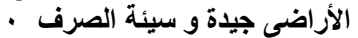

\begin{tabular}{|c|c|c|c|c|c|}
\hline & \multicolumn{2}{|c|}{$\begin{array}{c}\text { Levene's Test for } \\
\text { Equality of Variances }\end{array}$} & \multicolumn{3}{|c|}{$\begin{array}{c}\text { T-Test for Equality of } \\
\text { Means }\end{array}$} \\
\hline & F & Sig. & t & df & $\begin{array}{c}\begin{array}{c}\text { Sig. } \\
(2-\text { tailed) }\end{array} \\
\end{array}$ \\
\hline $\begin{array}{l}\text { Equal variances } \\
\text { assumed }\end{array}$ & 0.022 & 0.884 & 3.691 & 79 & .000 \\
\hline $\begin{array}{l}\text { Equal variances } \\
\text { not assumed }\end{array}$ & & & 3.700 & 78.397 & .000 \\
\hline
\end{tabular}

الحيويـة بالجمعيـات الزر اعيـة ، و عمل حقول إرشـادية لبيـان تأثثر هـاو

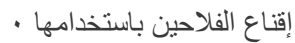

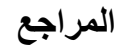

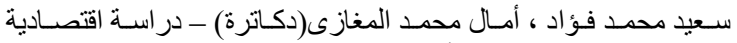

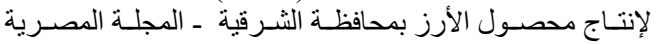

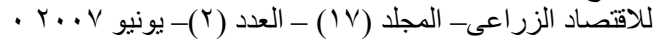

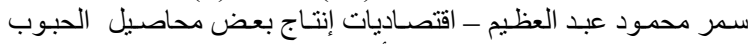

تحت الظروف البيئية في الأراضي الجديدة - رسـالة دكتور الهاه -

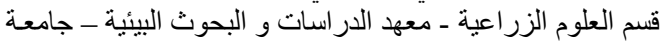

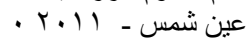

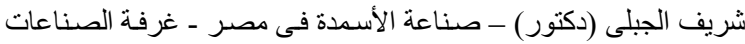

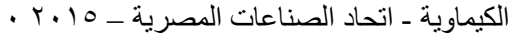

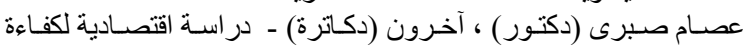

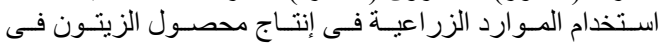

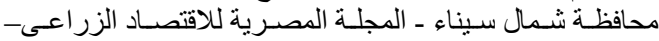

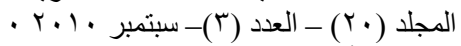

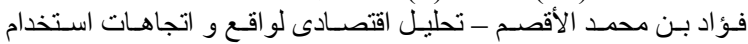

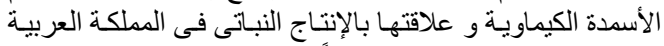

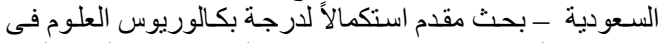

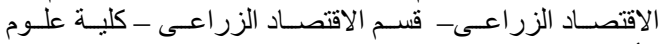

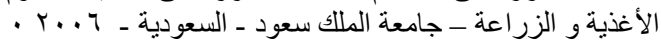

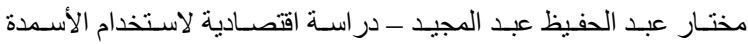

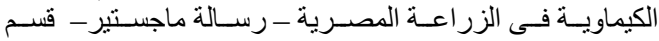

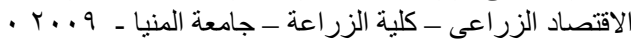

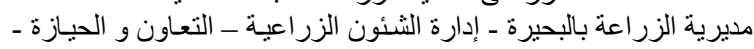

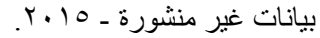

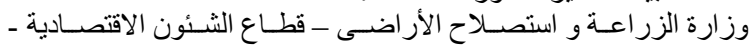

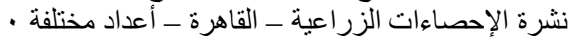

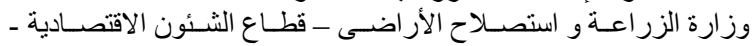

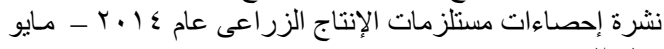
r. 10

Awudu Abdulai \& Wallace Huffman - Structural Adjustment and Economic Efficiency of Rice Farmers in Northern Ghana - Economic Development and Cultural Change - Vol. 48, No. 3 (April 2000).

Le Quang Long, others - Study on Economic Efficiency in Rice Production of Cuu Long Delta - Cuu Long Delta Rice Research Institute -Vietnam, Vol. $19-2013$.

Souleymane Ouedraogo (dr) - Technical and Economic Efficiency of Rice Production on the Irrigated Plain of Bagre (Burkina Faso): A Stochastic Frontier Approach - Social Sciences Institute - Journal of Economics and Sustainable Development - Burkina Faso - Vol.6, No.14, 2015.

\section{الملحق}

جدول 1 ـ الأهمية النسبية لمراكز محافظة البحيرة من حيث المسـاحة

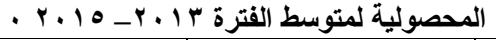

\begin{tabular}{|c|c|c|}
\hline الأهمية النسبية \% & المساحة المحصولية بالألف & البيان \\
\hline$r \cdot, \leqslant T$ & $r>7, \cdot \leq 9$ & أبو حمص \\
\hline $11,0$. & $1 \leqslant 9, \vee 1 V$ & كفر الدوار \\
\hline $1 \cdot, 9 V$ & $1 \leqslant Y, q 1 V$ & الدلنجات \\
\hline 9,7 & $1 r \leqslant, 999$ & كوم حماده \\
\hline $9, \leqslant 7$ & ITr,ITr & هور \\
\hline$r \Lambda_{,}, \varepsilon$ & $\leq 90, r 99$ & مر اكز أخرى \\
\hline $1 \ldots$ & $M \cdot r, \| r$ & الاجمالى \\
\hline
\end{tabular}

التعاون و الحيازة- بيانات غير منشورة 


\begin{tabular}{|c|c|c|c|c|c|}
\hline & \multicolumn{2}{|c|}{$\begin{array}{l}\text { Levene's Test for } \\
\text { Equality of } \\
\text { Variances }\end{array}$} & \multicolumn{3}{|c|}{$\begin{array}{c}\text { T-Test for } \\
\text { Equality of } \\
\text { Means }\end{array}$} \\
\hline & $\mathbf{F}$ & Sig. & $\mathbf{t}$ & df & $\begin{array}{c}\text { Sig. } \\
\text { (2- tailed) }\end{array}$ \\
\hline $\begin{array}{l}\text { Equal variances } \\
\text { assumed }\end{array}$ & 5.826 & 0.018 & -5.740 & 79 & .000 \\
\hline $\begin{array}{l}\text { Equal variances } \\
\text { not assumed }\end{array}$ & & & -5.614 & 64.528 & .000 \\
\hline
\end{tabular}

\begin{tabular}{|c|c|c|c|c|c|}
\hline & \multicolumn{2}{|c|}{$\begin{array}{l}\text { Levene's Test } \\
\text { for Equality of } \\
\text { Variances }\end{array}$} & \multicolumn{3}{|c|}{$\begin{array}{l}\text { T-Test for } \\
\text { Equality of } \\
\text { Means }\end{array}$} \\
\hline & $\mathbf{F}$ & Sig. & $\mathbf{t}$ & df & $\begin{array}{c}\text { Sig. } \\
\text { (2- tailed) }\end{array}$ \\
\hline $\begin{array}{l}\text { Equal variances } \\
\text { assumed }\end{array}$ & 8.276 & 0.005 & -1.969 & 79 & .052 \\
\hline $\begin{array}{l}\text { Equal variances } \\
\text { not assumed }\end{array}$ & & & -2.019 & 72.829 & .047 \\
\hline
\end{tabular}

\section{An economic study of the efficiency of the usage of chemical fertilizers in the production of the most important summer crops in Beheira Governorate Rania A. Elshaer Senior Res. Agricultural Economics Res. Instit., Agric.Res.Center}

\section{ABSTRACT}

This research aims mainly to identify the effect of the chemical fertilizers usage efficiency on rice crop productivity, and also the effect of agricultural drainage problems on the efficiency of the fertilizers usage and rice crop productivity in Beheira Governorate, as well as to determine the amount of wastage in chemical fertilizer usage. In addition to that the research aims to determine the economic phase of chemical fertilizers production of rice, as well as to identify the problems and obstacles faced by the farmers in the usage of chemical fertilizers and affect the productivity of rice crop so that the policy makers can put clear policies to increase the efficiency of the distribution and usage of this important Egyptian agricultural input, as well as to identify the extent of the growers knowledge and usage of organic and bio- fertilizers . The research shows that rice accounts for about $35 \%$ of the total area of summer crops cultivated in Beheira during the period $(2011-2015)$ and it also shows the decreasing of rice productivity in Beheira during the period $(2006-2015)$. The research results show that the amount of nitrogenous fertilizer units which achieve the maximum productivity of rice in good and bad drainage soils is more than the estimated amount of nitrogen fertilizer units by about $(44.5-144.3) \%$, and more than the amount delivered to farmers from the Ministry of Agriculture by about (72.5-191.7) \%.The amount of phosphate fertilizer which achieves the maximum productivity of rice in good and bad drainage soils is also more than the estimated amount of phosphate fertilizer units by about $(108-136) \%$. The research results also show that the nitrogenous and phosphate fertilizer productivity of rice in good drainage soils located in the third stage of production and that means that the production is decreasing for the negative marginal productivity of these elements, however in bad agricultural drainage soils these elements productivity located in the second stage of production .The research has estimated the wastage in chemical fertilizers used in the study sample in good drainage soils ranges from about $(32.26$ - 36.66) $\mathrm{kg}$ nitrogen unit, and about $(9.41$ - 11.34) $\mathrm{kg}$ Unit phosphate, however the wastage in chemical fertilizers used in the study sample in bad agricultural drainage soils ranges from about (12.81 - 14.23) kg nitrogen unit, and about (5.19 - 6.11) kg phosphate Unit, this is due to the need of bad agricultural drainage soils for larger quantities of fertilizers in order to achieve good productivity. The research shows that rice farmers in good agricultural drainage soils can increase their production by about (32 - 39.9) \% , and rice farmers in bad agricultural drainage soils can increase their production by about (10.7 to 13.8$) \%$ without any increase in the amount of chemical fertilizers used, that means that there is wastage in the usage of chemical fertilizers, which leads to lower nitrogen and phosphate unit productivity of rice in those soils as well as the unjustified increase of production costs. The study recommends the need to eliminate agricultural drainage problems, the need for strict control on fertilizers trade. In addition to that the government must overcome all obstacles to farmers at time of the receipt of agricultural crops and determine appropriate prices for these crops to stop traders monopoly of agricultural crops and control of their prices. as well as providing rice straw compressors and collecting the straw from the farmers without money instead of burning it . The study also recommends the need to organize guiding courses to educate farmers and listen to their problems and guide them continuously, as well as the need to provide organic, compound and bio-fertilizers in agricultural associations, prepare indicative fields to persuade farmers with these fertilizers benefits.

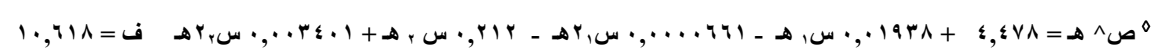

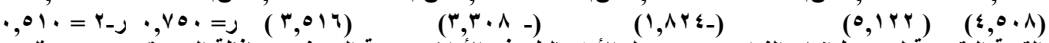

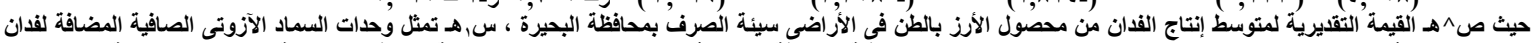

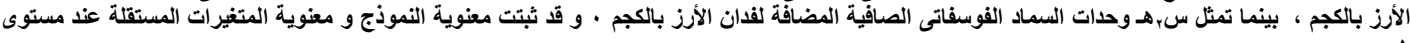

